\title{
The ancestors of the Earth's thermostat or how to link the rocks with the sky. \\ JÉRÔME GAILLARDET ${ }^{1}$ AND MATTHIEU EMMANUEL GALVEZ $^{2}$ \\ ${ }^{1}$ Institut de Physique du Globe de Paris \\ ${ }^{2} \mathrm{ETH}$
}

Presenting Author: gaillardet@ipgp.fr

In the middle of the $18^{\text {th }}$ century the idea that «fixed air» or «carbonic acid», known today as carbon dioxide was describing a «rotation» being continuously exchanged between the air, the plants, the animal and the rocks, emerged. The statement appeared far before it was realized that $\mathrm{CO}_{2}$ was eventually a green house gas having a an effect on climate. It can even be said that the discovery of the $\mathrm{C}$ cycle is consubstantially linked to the discovery of fixed air.

In this presentation, we will show how the discovery of the today so called "long term carbon cycle" (or geological) had not been separated initially from the discovery of the so called "short term carbon cycle". The discovery that photosynthesis and respiration were balancing mechanisms maintaining the habitability the atmosphere is concomitant to the idea that chemical weathering and volcanic activity were also key processes in the sustainability of life forms on the planet. We will, in particular show, how the progresses made by analytical chemistry in the first part of the $19^{\text {th }}$ century, in a context of strong competition between the nations of Western Europe, led to the discovery that the alteration of minerals at the Earth's surface could be seen as a neutralization reaction of atmospheric carbon dioxide, producing bicarbonate ions in solution and a solid residue symbolized by kaolin, an important economical resource at this period. The contributions of Ebelmen, Fournet in France or Bischof and Gehlen in Germany are particularly important and complementary with this regards.

We will finally pay attention to the way these new knowledges was transmitted (or not) to the following generations and then became extinct for more than a century. 